\title{
Vorwort: Europa über die Krise hinaus denken
}

\author{
Thomas Bagger und Mathias Jopp*
}

Wer heute nach dem ,Wert Europas' fragt, der meint in der Regel: Lohnt es die Anstrengungen, die uns die Bewältigung der Schuldenkrise abverlangt? Wir dürfen unseren Blick auf Europa aber nicht auf die Zumutungen der Krise verengen. Wir müssen Europa über die Krise hinaus denken - das wird uns helfen, die aktuelle Krise richtig einzuordnen, es wird uns auch helfen, die richtigen Schritte zu ihrer Überwindung zu tun.

Nur wenn das europäische Projekt seine Bürger überzeugt, wird neues Vertrauen entstehen. Dieses Vertrauen in Europa hat in den letzten Jahren empfindlich gelitten. Viele Menschen in Deutschland zweifeln an der Zukunftsfähigkeit der Währungsunion. Sie zweifeln daran, dass deutsches und europäisches Interesse noch deckungsgleich sind. Gleichzeitig sind bei einigen unserer Nachbarn Zweifel an der europäischen Ausrichtung Deutschlands aufgekommen. Worte aus Deutschland werden auf die europäische Goldwaage gelegt. Populisten erhalten Zulauf. Beunruhigende Rufe nach Renationalisierung werden laut. Um diese Erosion des Vertrauens in Europa aufzuhalten und umzukehren, bedarf es einer zeitgemäßen Begründung des europäischen Projekts.

Worin der Wert Europas liegt, lässt sich nicht aus Brüssel oder Berlin verlautbaren oder verordnen. Überzeugen wird nur eine Begründung, die aus einer lebendigen Debatte heraus entsteht. Zu dieser Debatte wollen der Planungsstab des Auswärtigen Amts und das Institut für Europäische Politik einen Anstoß geben. Deshalb haben wir die Frage nach dem Wert Europas im Januar 2012 auf die Tagesordnung eines gemeinsamen interdisziplinären Workshops im Auswärtigen Amt gesetzt. Dabei war unser Anliegen, die Debatte nicht auf das Finanzielle zu verengen. Deshalb lauteten unsere Leitfragen: Welchen materiellen und immateriellen Wert hat die europäische Einigung? Welche Bedeutung kommt Europa zu, wenn es gilt, unter den Vorzeichen der Globalisierung Wertvorstellungen und Interessen zu behaupten? Welchen Preis hätte ,Nicht-Europa', also ein Erodieren des europäischen Projekts?

Die hier abgedruckten Beiträge geben wesentliche Argumentationslinien und Denkanstöße des Workshops wieder. So unterschiedlich ihre Blickwinkel auf Europa sind, so klar ist das große Bild, das sie zeichnen. Es ist das Bild eines Europas, das wir in Zukunft notwendiger brauchen als je zuvor, um unter den Bedingungen der Globalisierung die grundlegenden Werte, Interessen und Lebensformen zu behaupten, die uns verbinden. Zugleich entsteht das Bild eines Deutschlands, für das es ohne geeintes Europa keine gute Zukunft gibt. Daraus ergibt sich die Notwendigkeit, am Projekt Europa festzuhalten und es weiterzuentwickeln. Die Skizze eines derart neu begründeten Zukunftsprojekts Europa bietet der Beitrag von Bundesaußenminister Guido Westerwelle in diesem Band. Eine abschließende Antwort auf die Frage nach dem Wert Europas ist er nicht. Er ist vielmehr eine Einladung zur Debatte. Diese Debatte ist nicht allein mit guten Argumenten zu gewinnen; sie muss auch Emotionen der Europäer ansprechen.

* Dr. Thomas Bagger, Leiter des Planungsstabs, Auswärtiges Amt, Berlin. Prof. Dr. Mathias Jopp, Direktor, Institut für Europäische Politik e.V., Berlin. 